\title{
Triplet state spectroscopy of conjugated polymers studied by pulse radiolysis
}

\author{
A.P. Monkman ${ }^{\mathrm{a},{ }^{*}}$, H.D. Burrows ${ }^{\mathrm{b}}$, M. da G. Miguel $^{\mathrm{b}}$, I. Hamblett ${ }^{\mathrm{c}}$, S. Navaratnam ${ }^{\mathrm{c}}$ \\ ${ }^{a}$ Department of Physics, University of Durham, Durham, UK \\ ${ }^{\mathrm{b}}$ Department of Chemistry, University of Coimbra, 3049 Coimbra, Portugal \\ ${ }^{\mathrm{c}}$ Paterson Institute for Cancer Research, Christie Hospital, Manchester, UK
}

\begin{abstract}
Using the technique of pulse radiolysis we have elucidated the energies and kinetics of triplet states in soluble luminescent conjugated polymers. Using poly(2-methoxy,5-(2'-ethyl-hexoxy)-p-phenylenevinylene) MEH-PPV as an example we explain this technique and show how it can be used to study the triplet states in conjugated polymers. Triplet energy transfer is used to determine $1^{1} A_{g}-1^{3} B_{u}$ energy gaps and the kinetics of triplet-triplet absorption yields triplet lifetimes. In the case of MEH-PPV, at concentrations up to $50 \mathrm{mg} / \mathrm{l}$, the triplet decay rate shows no change, indicating self-quenching of triplets is not significant. However, if very high electron beam doses are used, high intra chain triplet concentrations can be generated. In this high concentration regime triplet-triplet annihilation becomes effective, as determined by the onset of delayed fluorescence. (C) 2001 Elsevier Science B.V. All rights reserved.
\end{abstract}

Keywords: Conjugated polymers; Pulse radiolysis; Triplet state spectroscopy

\section{Introduction}

Luminescent conjugated polymers form a new technologically important class of materials to be used in light emitting display devices for the next generation of IT based consumer products $[1,2]$. Their photophysical properties are complex, with many different excited states having been observed over various time domains [3,4]. However, the nature and photophysics of triplet excitons in these materials is less well characterised. Their presence not only reduces luminescence quantum efficiency but also seriously limits the electroluminescence efficiency polymeric LEDs. Further, with the demonstration of optically pumped amplified spontaneous emission (ASE) the possibility of an all polymeric laser is not too far off. For these applications to be realised it is vital that the triplet states in these polymers are known and well characterised, particularly with respect to their absolute energies, degree of localisation, mobility and ability to energy transfer to oxygen form the highly reactive singlet oxygen. However, all known metal-free luminescent conjugated polymers have singlet ground states, such that direct optical excitation to the lowest triplet level has a vanishingly small transition probability. To provide unam-

\footnotetext{
${ }^{*}$ Corresponding author. Fax: +44-191-374-3848.

E-mail address: a.p.monkman@durham.ac.uk (A.P. Monkman).
}

biguous information on triplet states, optical studies on these systems need to be complemented by results obtained from other techniques. To date, optical excitation techniques have only allowed triplet-triplet transitions to be inferred, either by the long lifetime of the excited state as seen in photoinduced absorption [5-7] or from the modulation of the optical properties such as fluorescence by a magnetic field, as observed in various optically detected magnetic resonance measurements [8-10]. It is widely accepted from these measurements however, that triplet states are the cause of long lived excited states in conjugated polymers. To gain a full understanding of the electronic structure of these conjugated polymers and allow theoretical models to be refined it is important that the absolute energy of the triplet states, i.e. the $1^{1} A_{g}-1^{3} B_{u}$ energy separation is measured accurately. Phosphorescence in a conjugated polymers has been reported by Xu and Holdcroft, using the technique of upper excited state transfer (UEST) in poly(3-hexylthiophene) $[11,12]$ however, these initial measurements have not been reproduced. Other classical methods which have been used to induce $1^{1} A_{g}-1^{3} B_{u}$ absorption (and phosphorescence) such as heavy atom perturbation, using xenon, oxygen or zeolyte host matrices, are not compatible with polymeric materials as it is very difficult to co-evaporate or dissolve high molecular weight polymer with the host. Thus, we have turned our attention to a well-known, non-optical excitation technique to elucidate this problem, namely pulse radiolysis. 
From the classic work of Terenin and Ermolaev [13], triplet-triplet energy transfer has become one of the most important methods of specifically generating triplet states of organic molecules. Coupling this with pulse radiolysis of solutes in organic solvents provides an excellent technique for the selective creation and study of both excited states and charged species [14]. An intense, short-lived burst of highenergy radiation is passed through the sample, and the resultant non-equilibrium system studied by optical absorption spectroscopy, or some other analytical technique, analogous to photoinduced absorption. The radiation chemistry of solutions is dominated by the most prevalent species in solution, the solvent [15]. The primary process involves ionisation which in aromatic solvents such as benzene, is rapidly ( $<1 \mathrm{~ns})$ followed by charge recombination, leading to excited triplet and singlet states in the ratio $2.5: 1$ $\left(G_{\text {triplet }}=4.2, G_{\text {singlet }}=1.6\right.$, where $G$ is the number of excited states produced per $100 \mathrm{eV}$ of radiation absorbed [16]). By using appropriate energy acceptors, having lower triplet energies than benzene, short-lived singlet excited states and high $S_{1} \rightarrow T_{1}$ intersystem crossing efficiencies (such as biphenyl, $\tau_{\mathrm{f}}=16.0 \mathrm{~ns}, \Phi_{\mathrm{T}}=0.84$ [17]), Bensasson and Land have shown [18] that the excited triplet state of this acceptor $\left({ }^{3} \mathrm{~A}^{*}\right)$ could be selectively produced, and specifically populate the triplet state of a molecule of interest (S), through the reaction scheme

$\mathrm{Bz}+\mathrm{e}^{*} \rightarrow{ }^{1} \mathrm{Bz}^{*}+{ }^{3} \mathrm{Be}^{*}$

${ }^{1} \mathrm{Bz}^{*} \rightarrow{ }^{3} \mathrm{Bz}^{*}$

${ }^{3} \mathrm{Bz}^{*}+\mathrm{A} \rightarrow \mathrm{Bz}+{ }^{3} \mathrm{~S}^{*}$

${ }^{3} \mathrm{~A}^{*}+\mathrm{S} \rightarrow \mathrm{A}+{ }^{3} \mathrm{~S}^{*}$

subjected to the kinetically demanded concentration ratio $[\mathrm{Bz}] \gg[\mathrm{A}] \gg[\mathrm{S}]$. This concentration gradient, combined with diffusion controlled (solution) collisional triplet energy transfer ensures that only triplet energy is transferred down to the molecule under investigation. Triplet acceptors are chosen to have short singlet lifetimes and high intersystem crossing efficiency, so that only triplet states can be populated by triplet-triplet energy transfer from the acceptors. Transient absorption spectra of these states are of the pure triplets, and are free from contributions from singlet states or charged species. The energetics of this technique are shown schematically in Fig. 1. The experiment itself is quiet simple. Solutions of $S$ are made up in benzene with acceptors A, of different triplet energies (see Table 1). These have well characterise $T_{1}$ states, usually measured via phosphorescence. After rigorous degassing, radiolysis measurements are made to determine if triplet-triplet absorption can be seen from the sample $S$, indicating that the acceptor has transferred triplet energy to $S$, implying that $S$ has a low lying triplet state than A. When $E_{\mathrm{TA}}<E_{\mathrm{TS}}$, no induced triplet absorption is observed. For very accurate determination of the triplet energy of $S$, an acceptor who's triplet energy lies very close to that of the sample is required. In this

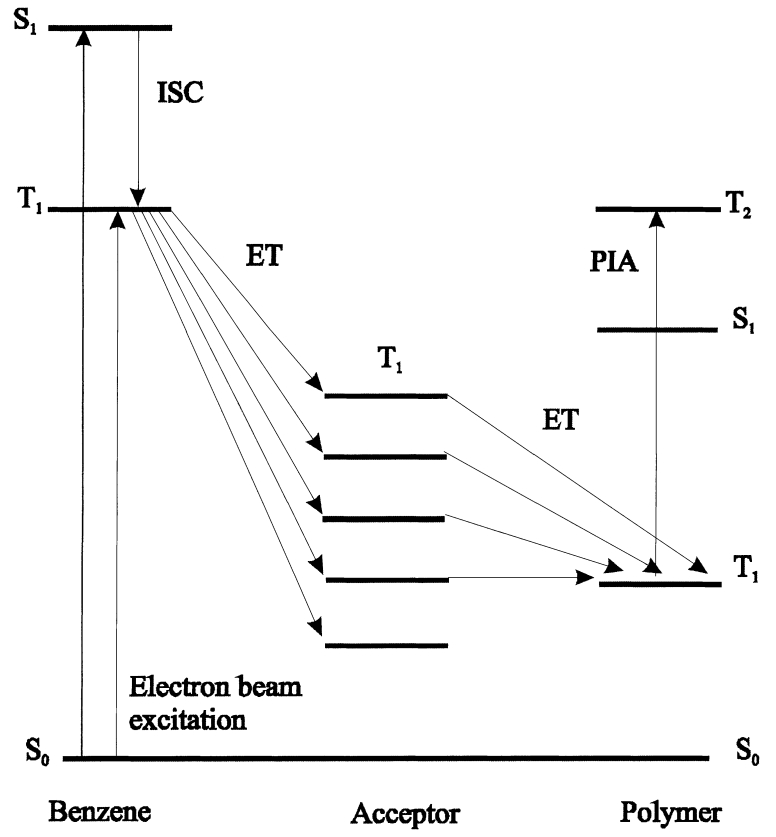

Fig. 1. Schematic of the triplet energy transfer process in pulse radiolysis, from solvent to acceptor to the molecule under investigation.

case triplet energy can be passed both from $A$ to $S$ and $S$ back to A, i.e. a pseudo equilibrium can be set up. In this case the kinetics of the energy transfer process changes markedly. By changing the relative concentrations of $\mathrm{A}$ and $\mathrm{S}$, precise determinations of $E_{\mathrm{TS}}$ can be made. Such energy transfer experiments have been performed on MEH-PPV [20] and a range of other soluble conjugated polymers [21] in benzene solutions using a range of triplet acceptors.

Pulse radiolysis of solutions was made using 1-200 ns pulses (0.2-3.5 nc doses) from the $10 \mathrm{MeV}$ electron linear accelerator of the Paterson Institute for Cancer Research Free Radical Research Facility (Manchester, UK). Transient absorption and bleaching detection can be made either spectroscopically using a grating monochrometer and gated fibre coupled, Si-diode array, allowing submicrosecond time resolved spectra to be recorded. For accurate dynamics of

Table 1

Triplet energies of various organic molecules used as triplet energy acceptors in pulse radiolysis experiments ${ }^{\mathrm{a}}$

\begin{tabular}{lll}
\hline Acceptor & $\mathrm{kcal} / \mathrm{mol}[17]$ & $\mathrm{eV}$ \\
\hline Benzophenone & 68.6 & 2.97 \\
Biphenyl & 65.4 & 2.84 \\
Naphthalene & 60.5 & 2.63 \\
Terphenyl & 58.3 & 2.52 \\
Benzil & 53.4 & 2.32 \\
Anthracene & 42.3 & 1.84 \\
Perylene & 35.4 & 1.54 \\
meso-Tetrakis(phenyl)porphyrin & 33.0 & 1.43 \\
Tetracene & 29.2 & 1.27 \\
Rubrene & 26.3 & 1.14 \\
$\mathrm{O}_{2}\left({ }^{1} \Delta_{g}\right)$ & 21.7 & 0.94 \\
\hline \multicolumn{1}{c}{${ }^{\mathrm{a}}$ After [17]. } & &
\end{tabular}




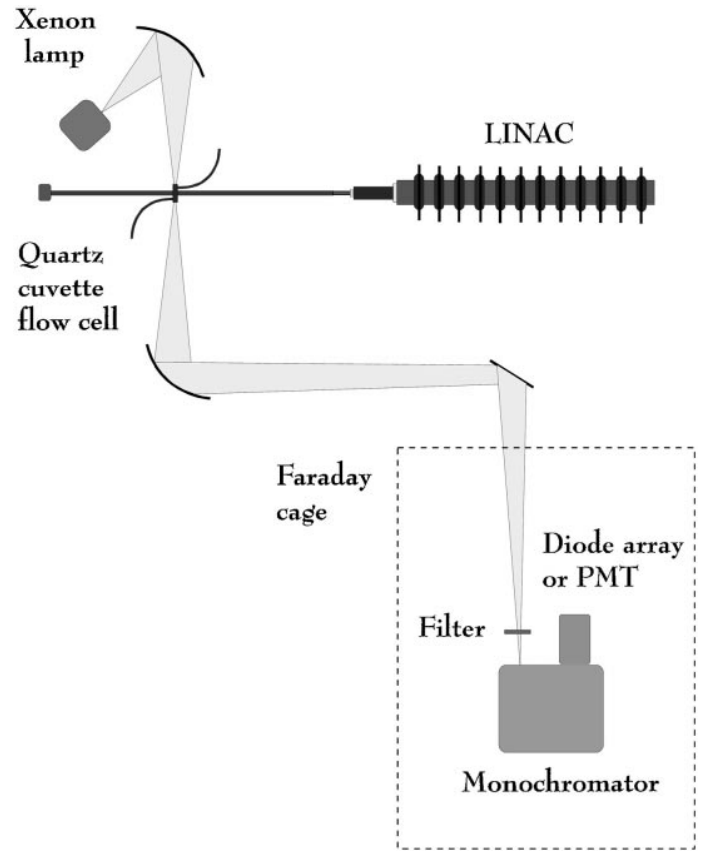

Fig. 2. Schematic of the experimental arrangement of the pulse radiolysis spectrometer at the Paterson Institute for Cancer Research Free Radical Research Facility.

signal build-in and decay, single wavelength measurements are made using fast photomultiplier tubes, Si or Ge diodes. A remotely controlled sample flow system is used to allow the solutions in the quartz sample flow cell to be replenished after every shot of the excitation beam. All solutions were prepared using polymer molar concentrations based on their repeat unit mass, not molecular weight. Once solutions had been prepared, they were degassed by bubbling with Ar for $30 \mathrm{~min}$. Flasks were transferred to the LINAC, and plumbed into the flow cell system whilst maintaining the integrity of the Ar atmosphere. To aid identification of various possible excited species, samples could be re-oxygenated by bubbling with oxygen, i.e. to quench triplet states were monitored by optical absorption spectroscopy using a xenon source and gated diode array detection. A schematic of the spectrometer is given in Fig. 2.

\section{Results and discussion}

A typical pulse radiolysis spectrum of Ar saturated solutions of biphenyl (0.1-0.01 M) MEH-PPV (0.625-200 mg/l) in benzene is shown in Fig. 3, with decay and build in kinetics in the inset. A transient absorption is observed at $3.4 \mathrm{eV}$. This is ascribed to the biphenyl triplet state $[18,19]$. Note also the bleaching of the biphenyl $S_{0} \rightarrow S_{1}$ transition at ca. $4.1 \mathrm{eV}$. The induced absorption centred at $1.5 \mathrm{eV}$, onset at ca. $1.4 \mathrm{eV}$, and bleaching at $2.4 \mathrm{eV}$ indicate triplet transfer to the MEH-PPV. The rate of grow-in of both these MEH-PPV features is identical, indicating that electrons are excited out of the MEH-PPV ground state (HOMO) to form a new

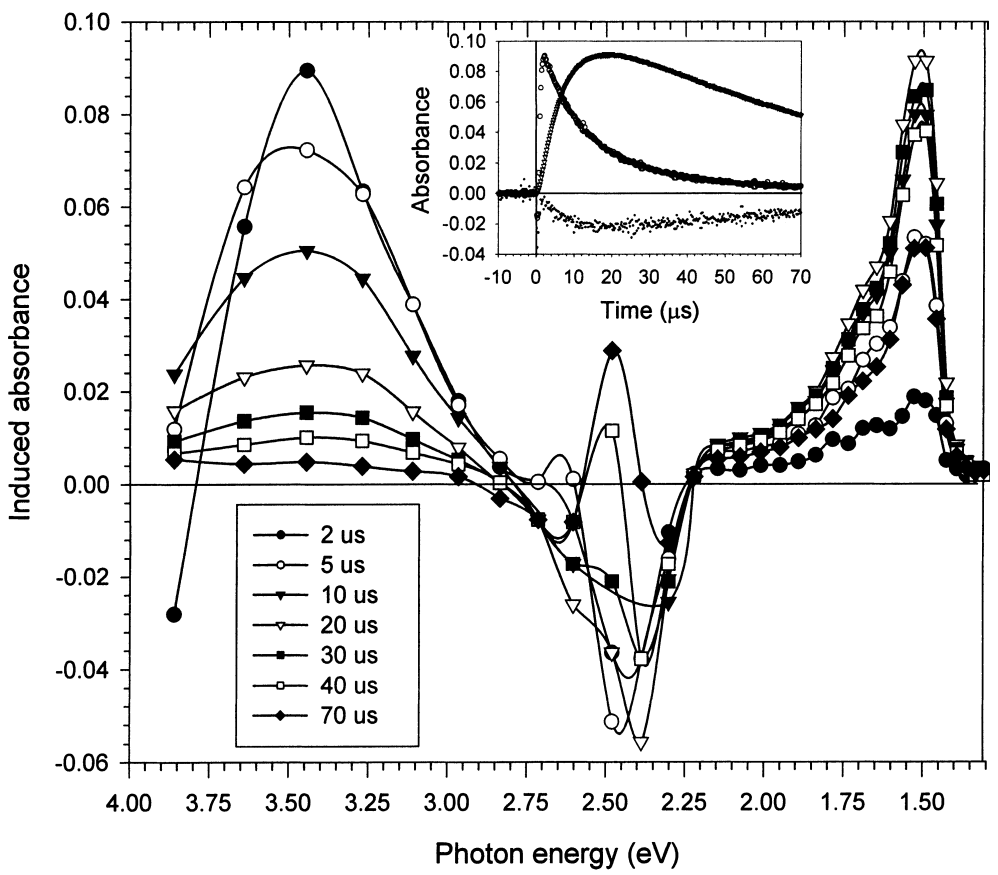

Fig. 3. Radiolysis spectrum of MEH-PPV in benzene with biphenyl triplet acceptor. Induced absorption at $3.4 \mathrm{eV}$ which decays rapidly is the triplet-triplet absorption of the biphenyl. Bleaching of the MEH-PPV singlet ground state is observed at $2.4 \mathrm{eV}$. The peak of the triplet-triplet absorption in the MEH-PPV is observed at $1.5 \mathrm{eV}$. This absorption also shows evidence of resolvable vibronic structure. Inset shows the kinetics of the three main features observed in the radiolysis experiment, namely biphenyl triplet absorption at $3.4 \mathrm{eV}(\mathrm{O})$, MEH-PPV singlet bleaching at $2.4 \mathrm{eV}(+)$ and triplet absorption at $1.5 \mathrm{eV}(\nabla)$. The rate of decay of the biphenyl triplet is equal to the grow-in rate of both the MEH-PPV features. Both the MEH-PPV bleaching and triplet absorption grow-in at the same rate indicating triplets are formed from the singlet ground state. 
occupied state, optical excitation which we observe at $1.5 \mathrm{eV}$. The grow-in rate parallels the rate of decay of the biphenyl triplet indicating transfer of triplets from the biphenyl to the MEH-PPV, (see Fig. 3 inset). Only very small levels of directly induced absorption between benzene and MEH-PPV was detected at the highest concentrations used. The induced optical absorption in the MEH-PPV arising from direct triplet transfer is identical to that observed in PIA experiments on thin films of the polymer $[5,7]$. There is only a very small energy shift ca. $100 \mathrm{meV}$, between the solution and solid state features. This indicates the localised nature of the triplet states and that the species giving rise to this induced absorption are neutral as they show very little solvatochromic shift. It also clearly shows that the triplet states are more localised than singlet states as previous suggested [8,9], and that interchain interactions cause little perturbation to the triplet states. We find that situations holds true for over 10 conjugated polymers we have studied so far using radiolysis.

We have determined the $1^{1} A_{g}-1^{3} B_{u}$ energy separation using the energy transfer technique in MEH-PPV, and many other soluble conjugated polymers. In the case of MEH-PPV we find that sensitisers with triplet energies $=1.54 \mathrm{eV}$ (perylene) will transfer triplet energy efficiently to $\mathrm{MEH}$ PPV. Pulse radiolysis of a solution of biphenyl $(0.1 \mathrm{M})$ and MEH-PPV (25 mg/l) in benzene in the presence of varying concentrations of rubrene $\left(E_{\mathrm{T}}=1.14 \mathrm{eV}\right)$ shows efficient quenching of the MEH-PPV triplet state, with a rate constant of $7.5 \times 10^{9} \mathrm{M}^{-1} \mathrm{~s}^{-1}$, typical of triplet-triplet energy transfer in organic molecules. Pulse radiolysis of solutions of biphenyl (0.1 M), MEH-PPV (200 mg/l) tetracene $(1 \mathrm{mM})$ in benzene showed formation of both tetracene and $\mathrm{MEH}$ PPV triplet states. Although it was not possible to set up an equilibrium between them, due to solubility problems, the fact that ${ }^{3} \mathrm{MEH}-\mathrm{PPV}^{*}$ decay is not affected by tetracene $\left(E_{\mathrm{T}}=1.27 \mathrm{eV}, \tau_{\text {singlet }}=6.4 \mathrm{~ns}, \Phi_{\mathrm{T}}=0.62\right.$ [17] $)$ suggests that its energy must be equal to or just below that of tetracene. Thus, we estimate that the energy of the first triplet level lies $1.27 \pm 0.07 \mathrm{eV}$ above the (singlet) ground state.

Up to a concentration of $50 \mathrm{mg} / \mathrm{l}$, the decay rate of the MEH-PPV induced absorption, $\tau=100 \mu$ s (a lower limit is given, as the observed decay may be governed by impurity quenching) was found to be independent of MEH-PPV concentration, indicating that up to this concentration range self-quenching of the triplet state is not significant. However, when high electron beam doses are used, the number of triplets per chain increases markedly. The number of triplets transferred to the acceptor is unaffected by the increasing beam dose. However, when the MEH-PPV triplet decay kinetics are analysed we have to use a competing first and second order fit. The latter term corresponds to an intramolecular bimolecular decay. This we ascribe to triplet-triplet annihilation. To confirm that this is the case, we performed the radiolysis experiment at high doses, with the probe light turned off and monitored any emission from the solutions.

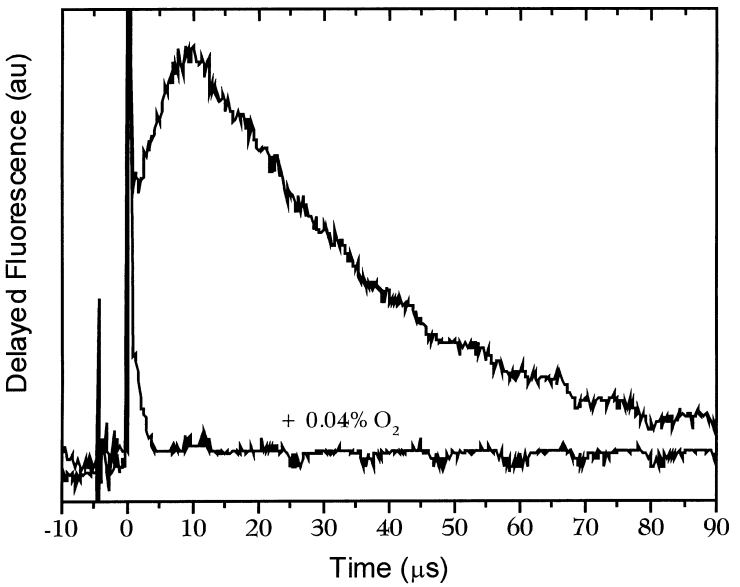

Fig. 4. The time dependence of the DF signal, measured at $2.23 \mathrm{eV}$, from an MEH-PPV solution. The effect of addition of $0.04 \% \mathrm{O}_{2}$ can clearly be seen.

Fig. 4 shows a typical emission decay monitored at $2.23 \mathrm{eV}$ $(555 \mathrm{~nm})$, near the peak of the $1^{1} B_{u}-1{ }^{1} A_{g}$ emission from MEH-PPV. After the electron beam pulse we observe the initial build up of the delayed fluorescence (DF) and subsequent decay. The DF has a lifetime of $36 \mu$ s (at $0.2 \mathrm{nc}$ dose rate). The measured kinetics of the DF build up matches that of the triplet-triplet absorption in the MEH-PPV, and can be seen in the Fig. 4, the DF signal is completely quenched by the addition of $0.04 \%$ oxygen to the solution. This is clear evidence that the DF signal comes from excited state singlet production as a consequence of triplet pair decay, i.e. triplettriplet annihilation

$\mathrm{T}_{1}+\mathrm{T}_{1} \leftrightarrow{ }^{1,3,5}\left(\mathrm{~T}_{1} \ldots \mathrm{T}_{1}\right) \leftrightarrow\left\{\begin{array}{l}\mathrm{S}_{0}+\mathrm{T}_{n} \\ \mathrm{~S}_{0}+\mathrm{S}_{n}^{*}\end{array}\right.$

In the case of this type of radiolysis measurement, there is a clear advantage over optical excitation. Here we can transfer many triplet states to each polymer chain in solution at the highest doses rates we have used, we estimated that order of 30 triplets per chain can be transferred before saturation. Thus, it is relatively easy to observed DF from triplet-triplet annihilation. In the case of optical excitation, we have previously shown that the ISC yield for the process, $1^{1} A_{g}-1^{3} B_{u}$ is very low, of order $2 \%$ [20] thus, it is very difficult to build up a high enough triplet population on a chain to observe DF [22]. From the kinetics of the DF, which we have observed in several other polymers also, it is clear that the intra chain hopping rate for triplets is fairly low. We shall comment more on this in the near future.

Thus, we have found that pulse radiolysis is an excellent technique for studying the triplet states in soluble conjugated polymers. It offers a practical method by which the $1^{1} A_{g}-1^{3} B_{u}$ energy separation can be measured in polymers with vanishingly small phosphorescence and ISC yields. Further, as we can generate high triplet concentrations on each polymer chain we can study triplet-triplet interactions including annihilation leading to DF. In related work, we 
also have shown that radiolysis can be used to study spectroscopically and kinetically the positive and negative charged states in conjugated polymers [23]. Combining all results from these experiments allows unparalleled identification of the spectroscopic signatures of the excited states of these materials.

\section{Acknowledgements}

We would like to acknowledge the EPSRC (GR/ M86040), the British council/CRUP (project B-9/97). Covion for their very kind supply of MEH-PPV. The Paterson Institute for Cancer Research Free Radical Research Facility (Manchester, UK) for access to the pulse radiolysis spectrometer, Dr. Donald Allan for all his help and Scottish humour.

\section{References}

[1] J.H. Burroughes, D.D.C. Bradley, A.R. Brown, R.N. Marks, R.H. Friend, P.L. Burn, A.B. Holmes, Nature 347 (1990) 539.

[2] R.H. Friend, R.W. Gymer, A.B. Holmes, J.H. Burroughes, R.N. Marks, C. Taliani, D.D.C. Bradley, D.A. dos Santos, J.L. Bredas, M. Logdlund, W.R. Salaneck, Nature 397 (1999) 121.

[3] N.T. Harrison, G.R. Hayes, R.T. Phillips, R.H. Friend, Phys. Rev. Lett. 77 (1996) 1881
[4] S.V. Frolov, M. Ozaki, W. Gellermann, K. Yoshino, Z.V. Vardeny, Phys. Rev. Lett. 78 (1997) 729.

[5] R.H. Friend, D.D.C. Bradley, P.D. Townsend, J. Phys. D: Appl. Phys. 20 (1987) 1367.

[6] H.S. Woo, S.C. Grahem, D.A. Haliday, D.D.C. Bradley, R.H. Friend, P.L. Burn, A.B. Holmes, Phys. Rev. B 46 (1992) 7379.

[7] X. Wei, B.C. Hess, Z.V. Vardeny, F. Wudl, Phys. Rev. Lett. 68 (1992) 666.

[8] L.S. Swanson, J. Shinar, K. Yoshino, Phys. Rev. Lett. 65 (1990) 1140.

[9] L.S. Swanson, P. Lane, J. Shinar, F. Wudl, Phys. Rev. B 44 (1991) 10617.

[10] J. Shinar, L.S. Swanson, SPIE Proceedings 1910 (1993) 147.

[11] B. Xu, S. Holdcroft, J. Am. Chem. Soc. 115 (1993) 8447.

[12] B. Xu, S. Holdcroft, Adv. Mater. 6 (1994) 325

[13] A.N. Terenin, V. Ermolaev, Trans. Faraday Soc., 521042 (1956), and references therein.

[14] J.H. Baxendale, M.A.J. Rodgers, Chem. Soc. Rev. 7 (1978) 235.

[15] A.J. Swallow in: Farhataziz, M.A.J. Rodgers (Eds.), Radiation Chemistry, Principles and Applications VCH, Weinheim, 1987.

[16] J.H. Baxendale, M. Fiti, J. Chem. Soc., Faraday Trans. 268 (1972) 218.

[17] L. Murov, I. Carmichael, G.L. Hug, Handbook of Photochemistry, 2nd Edition, Marcel Dekker, New York, 1993.

[18] E.J. Land, Proc. Roy. Soc. A 305 (1968) 457.

[19] R. Bensasson, E.J. Land, Trans. Faraday Soc. 67 (1971) 1904.

[20] A.P. Monkman, H.D. Burrows, M.G. Miguel, I. Hamblett, S. Navaratnam, J. Chem. Phys. 307 (1999) 303.

[21] A.P. Monkman, H.D. Burrows, I. Hamblett, S. Navaratnam, submitted for publication.

[22] J. Partee, E.L. Frankevich, B. Uhlhorn, J. Shinar, Y. Ding, T.J. Barton, Phys. Rev. Lett. 82 (1999) 3673.

[23] H.D. Burrows, M.G. Miguel, A.P. Monkman, L.E. Horsburgh, I. Hamblett, S. Navaratnam, J. Chem. Phys. 112 (2000) 3082. 